\title{
Driving performance of retinitis pigmentosa patients
}

\author{
GERALD A. FISHMAN, ${ }^{1}$ ROBERT J. ANDERSON, ${ }^{2}$ \\ LARRY STINSON, ${ }^{1}$ AND AMANUL HAQUE ${ }^{1}$ \\ From the ${ }^{1}$ Department of Ophthalmology, University of Illinois Hospital Eye and Ear Infirmary, \\ and the ${ }^{2}$ Epidemiology and Biometry Program, School of Public Health, University of \\ Illinois at the Medical Center, Chicago, USA
}

SUMMARY The driving performance of 42 patients with retinitis pigmentosa (RP) was compared with that of 87 control subjects. Although the patients with RP were involved in more isolated road accidents than the control group, $50 \%$ were not involved in any accident over the most recent 5-year driving period. When the associations between driving performance and case/control status were examined (the number of driving hours per week and driving years being taken into account), differences in the number of accidents between the 2 groups were significant because of a disproportionately high number of accidents caused by a subgroup of female RP patients. No significant correlations were apparent between central visual efficiency or peripheral field efficiency and number of road accidents.

A consensus of opinion on the precise standards of visual performance necessary to operate a motor vehicle safely is lacking. We evaluated the driving performance, in terms of frequency of accidents, in 42 patients with retinitis pigmentosa (RP) who have varying degrees of central and peripheral field loss and compared it with 87 controls without ocular disease who were of similar age.

\section{Subjects and methods}

The 42 patients with RP were selected from a larger RP clinic population. All patients selected showed characteristic features of the disease, which included abnormal rod and cone function by electroretinography, peripheral field loss, bone spicule pigmentation, attenuated retinal vessels, and some degree of night blindness. Patients with a visual acuity of less than 20/100 in the best corrected eye and those who were aphakic were arbitrarily excluded.

All patients underwent a thorough ophthalmic examination, which consisted of best corrected visual acuity and peripheral field examination with a Goldmann perimeter, with a 4-e-II test target. Visual acuity examinations were then converted into central visual efficiency by using the Lebensohn

Correspondence to Dr G. A. Fishman, University of Illinois Hospital Eye and Ear Infirmary, 1855 W Taylor St, Chicago, IL 60612, USA. near-vision chart and data for central field efficiency 1 (Table 1). Peripheral field examinations were similarly converted into field efficiency by totalling the degree of visual field in eight meridians (horizontal, vertical, oblique $45^{\circ}$, and $225^{\circ}, 135^{\circ}$, and $315^{\circ}$ ) from the central fixation point and dividing by $5 .^{2}$ Dimensions of the total horizontal meridian field diameters for the $42 \mathrm{RP}$ patients are recorded in Table 2.

The 87 control subjects were from a population similar in age to the RP patients. They were selected from (1) family members of RP patients, (2) family members of patients seen for other ocular diseases by one of the authors (G.A.F.), (3) relatives of patients being seen in the general eye clinic, or (4) clerical staff members of the ophthalmology department. None of the control subjects showed any ophthalmic or general defects that might hinder the safe operation of a motor vehicle.

Each subject was interviewed by 2 of the authors (L.S. and A.H.). Specific information on the total number of driving years, usual driving hours per week, and involvement in moving collision violations was obtained. Accidents in which RP patients and controls were either cited or not cited as having caused the accident were included. The study period was arbitrarily chosen to be 5 years to facilitate reasonably accurate recall. The patients' most recent visual field and central acuity are also reasonably representative of their visual performance for this 
Table 1 Conversion of visual acuity to visual efficiency

\begin{tabular}{lllllllllll}
\hline Visual acuity (Snellen) & $20 / 20$ & $20 / 25$ & $20 / 30$ & $20 / 40$ & $20 / 50$ & $20 / 60$ & $20 / 70$ & $20 / 80$ & $20 / 100$ & $20 / 200$ \\
\hline Visual efficiency (\%) & 100 & 95 & 90 & 85 & 75 & 70 & 64 & 59 & 50 & 20 \\
\hline
\end{tabular}

Table 2 Total horizontal meridian degrees for peripheral visual field in 42 patients with retinitis pigmentosa

\begin{tabular}{|c|c|c|c|}
\hline Patient no. & Right eye & Left eye & Both eyes \\
\hline 1 & 30 & 15 & 45 \\
\hline 2 & 15 & 10 & 25 \\
\hline 3 & 20 & 20 & 40 \\
\hline 4 & 10 & 15 & 25 \\
\hline 5 & 15 & 15 & 30 \\
\hline 6 & 10 & 10 & 20 \\
\hline 7 & 10 & 20 & 30 \\
\hline 8 & 20 & 20 & 40 \\
\hline 9 & 10 & 20 & 30 \\
\hline 10 & 15 & 35 & 50 \\
\hline 11 & 20 & 20 & 40 \\
\hline 12 & 25 & 25 & 50 \\
\hline 13 & 25 & 20 & 45 \\
\hline 14 & 20 & 20 & 40 \\
\hline 15 & 15 & 20 & 35 \\
\hline 16 & 30 & 10 & 40 \\
\hline 17 & 20 & 15 & 35 \\
\hline 18 & 15 & 10 & 25 \\
\hline 19 & 15 & 35 & 50 \\
\hline 20 & 25 & 25 & 50 \\
\hline 21 & 25 & 20 & 45 \\
\hline 22 & 25 & 25 & 50 \\
\hline 23 & 30 & 35 & 65 \\
\hline 24 & 20 & 20 & 40 \\
\hline 25 & 20 & 30 & 50 \\
\hline 26 & 35 & 20 & 55 \\
\hline 27 & 30 & 45 & 75 \\
\hline 28 & 60 & 45 & 105 \\
\hline 29 & 35 & 35 & 70 \\
\hline 30 & 40 & 35 & 75 \\
\hline 31 & 25 & 50 & 75 \\
\hline 32 & 50 & 50 & 100 \\
\hline 33 & 40 & 45 & 85 \\
\hline 34 & 50 & 75 & 125 \\
\hline 35 & 20 & 45 & 65 \\
\hline 36 & 65 & 20 & 85 \\
\hline 37 & 60 & 60 & 120 \\
\hline 38 & 45 & 45 & 90 \\
\hline 39 & 105 & 50 & 155 \\
\hline 40 & 70 & 50 & 120 \\
\hline 41 & 70 & 70 & 140 \\
\hline 42 & 120 & 110 & 230 \\
\hline
\end{tabular}

5-year period. It is known by history that 31 of the RP patients voluntarily limited themselves exclusively to daytime driving.

\section{Results}

The 2 study groups were very similar in basic characteristics (Table 3 ).

Table 4 shows the cross-classification of number of accidents with case/control status. There was a statistical difference in the number of accidents experienced in the last 5 years between RP patients and controls $\left(\chi^{2}=5.58, p=0.02\right)$, with the number of accidents recorded as either 0 or $\geqslant 1$. During the 5 -year period $21(50 \%)$ of the RP patients were not involved in any motor vehicle accidents compared with $62(71 \%)$ of the controls. Table 5 indicates the association between the number of accidents in the past 5 years for RP patients and controls when comparisons are made after controlling for hours per week spent driving, driving years, and sex. Significant differences between the 2 groups exist $(p<0.05)$ for females and for those driving the least number of years. A difference close to statistical significance $(p=0.06)$ exists between the RP patients and controls when comparing those in each group who drove the least number of hours per week.

Table 3 Comparison of retinitis pigmentosa $(R P)$ patients (42) with controls (87)

\begin{tabular}{llll}
\hline & Controls & $R P$ \\
\cline { 1 - 2 } Sample size & 87 & 42 \\
Percent male & $44 \%$ & $52 \%$ \\
Mean age (yr) & 37 & 38 \\
Age limits (yr) & $21-72$ & $21-75$ \\
Mean years driving experience & $17 \cdot 2$ & 17.4 \\
\hline
\end{tabular}

Table 4 Accident records of retinitis pigmentosa $(R P)$ patients compared with those of controls for a 5-year period

\begin{tabular}{lllll}
\hline & \multicolumn{2}{l}{ No. of accidents } & & \\
\cline { 2 - 5 } \cline { 3 - 4 } & 0 & $\geqslant 1$ & \multicolumn{2}{c}{ Total } \\
\hline RP & 21 & 21 & 42 & $\chi^{2}=5.58$ \\
Control & 62 & 25 & 87 & $\mathrm{p}=0.02$ \\
\hline
\end{tabular}


Fig. 1 Driving records are correlated with peripheral visual field efficiency (average of 2 eyes) in 42 patients with retinitis pigmentosa. Black circles (O) indicate 1 to 10 driving hours per week; open circles (O) 10 to 15 hours per week; triangles $(\triangle) 15$ to 20 hours per week; and squares ( $\square$ ) more than 20 hours per week.

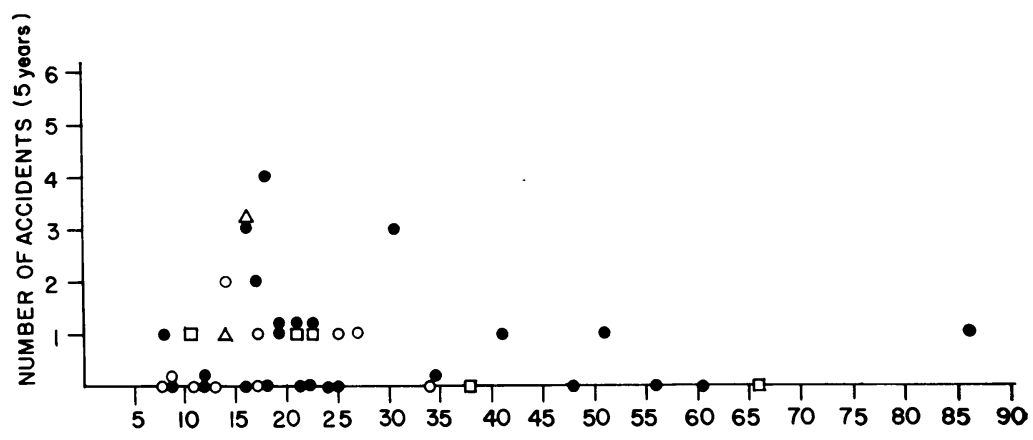

PERIPHERAL VISUAL FIELD EFFICIENCY

(overage of two eyes)

Table 5 Statistical differences in the number of accidents of retinitis pigmentosa patients compared with those of controls*

\begin{tabular}{lll}
\hline & $\chi^{2}$ & $p$ \\
\hline Driving hours/week & & \\
$1-10$ & 3.55 & 0.06 \\
$10-20$ & 3.23 & 0.14 \\
$20+$ & 0.65 & 0.42 \\
Years driving & & \\
$5-10$ & 7.84 & 0.005 \\
$11-20$ & 0.41 & 0.52 \\
$21+$ & 0.98 & 0.32 \\
Sex & & \\
Male & 0.06 & 0.81 \\
Female & 9.26 & 0.002 \\
\hline
\end{tabular}

*Comparisons are made for each category of driving hours/week, years driving, and sex.
Within the control group males were noted to have caused a significantly greater number of accidents than females $(42 \%$ versus $18 \%, \mathrm{p}=0.02)$. These findings are consistent with those of previous investigations on accident rates in the general population. ${ }^{3}$ Such differences were not found in the RP group, in which $45 \%$ of the males had one or more accidents compared with $55 \%$ of the females $(\mathrm{p}=0.54)$.

Fig. 1 shows no relationship $(r=-0 \cdot 13)$ between the peripheral visual field efficiency, averaged from both eyes, and the number of accidents. Similar results are seen when comparing central visual efficiency (averaged from both eyes) with the number of accidents (Fig. 2; $r=-0 \cdot 09$ ). Fig. 3 shows no relationship $(r=-0 \cdot 16)$ between the average of both peripheral and central efficiency and the number of accidents.

Further analysis of a comparison between RP
Fig. 2 Driving records are correlated with central visual efficiency (average of 2 eyes) in 42 patients with retinitis pigmentosa. See legend of Fig. 1 for key to symbols.

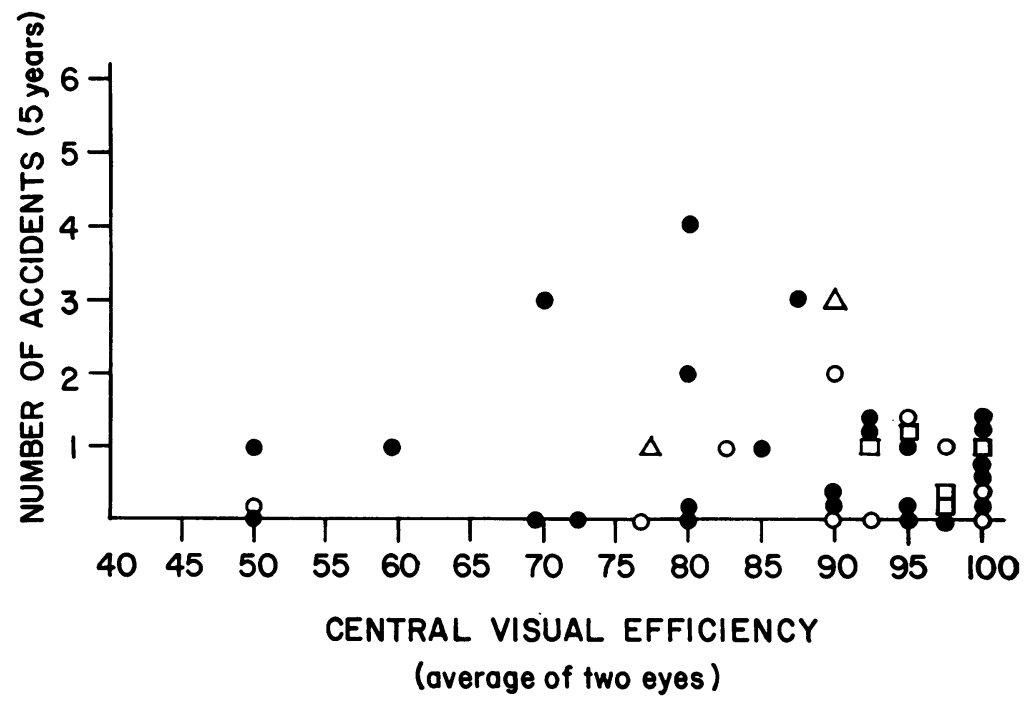




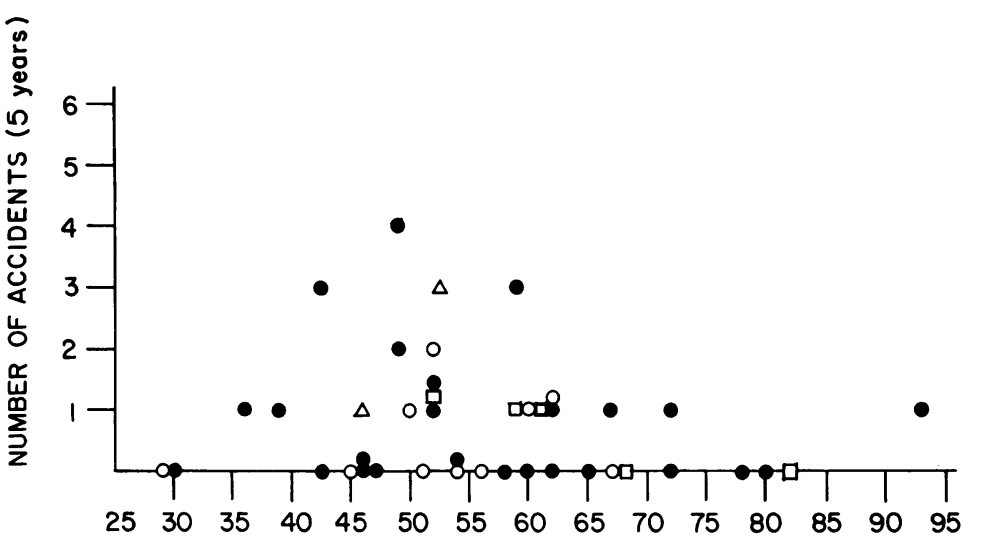

Fig. 3 Driving records are correlated with the average of central visual efficiency (average of 2 eyes) and peripheral visual field efficiency (average of 2 eyes) in 42 patients with retinitis pigmentosa. See legend of Fig. 1 for key to symbols.

AVERAGE OF CENTRAL VISUAL EFFICIENCY (overage of two eyes) AND PERIPHERAL FIELD EFFICIENCY (average of two eyes)

patients and controls by the Mantel-Haenszel ${ }^{4}$ statistic is shown in Table 6. A statistically significant difference in the number of accidents between the 2 groups existed only when females within each group were compared. There were 5 female RP patients who drove 5 to 10 years. Four of the 5 were under age 30 . All were involved in 1 or more accidents; 3 had 3 accidents and 1 had 4 accidents. Four of the 5 drove 1 to 10 hours per week, while 1 drove 15 to 20 hours per week. For reasons as yet unapparent a greater number of these RP females who drove 1 to 10 hours per week and from 5 to 10 years were involved in a greater number of accidents than a control female population who also drove the same number of hours per week and for the same number of years. This difference in the number of accidents was not apparent for RP females who drove for longer than 10 years or for RP males independent of their number of driving years. Table 7 shows that the greater incidence of accidents among RP females driving 5 to 10 years was not explicable by a significantly greater extent of central vision or peripheral field loss. Moreover, significant age differences between RP females and normal female controls who drove 5 to 10 years were not a factor, since the mean ages for the RP and control females were 31 years and 26 years, respectively.

\section{Discussion}

Previous authors have described the impact of ophthalmic disorders on driving performance, mostly in drivers with low vision who use telescopic lenses. ${ }^{5-8}$ Few data are available on the safety records of patients who have substantial restriction of peripheral visual field, though it has been indicated that restriction in this field of vision presents
Table 6 Mantel-Haenszel statistics* comparing number of accidents for retinitis pigmentosa patients with those of controls

\begin{tabular}{lll}
\hline Control variable & $\chi^{2}(M H)$ & $p$ \\
\hline Driving hours/week & 6.24 & 0.01 \\
Years driving & 6.14 & 0.01 \\
Sex & 4.99 & 0.03 \\
Driving hours/week and years driving & 7.21 & 0.007 \\
Sex and driving hours/week & 5.55 & 0.02 \\
Driving hours/week & & \\
$\quad$ Males only & 0.49 & 0.48 \\
$\quad$ Females only & 7.26 & 0.01 \\
Sex and years driving & 6.07 & 0.01 \\
Years driving & & \\
$\quad$ Males only & 0.12 & 0.73 \\
$\quad$ Females only & 10.52 & 0.001 \\
Sex, driving hours/week, and years driving & 6.74 & 0.009 \\
$\quad$ Males only & 0.53 & 0.47 \\
Females only & 8.90 & 0.003 \\
\hline
\end{tabular}

*Data were analysed by the Mantel-Haenszel statistic, $\chi^{2}(\mathrm{MH})$, which tests for the existence of an association between 2 variables (case/control status and number of accidents) while controlling for the effects of 1 or more confounding variables (e.g., years driving, sex, sex by years driving).

Table 7 Mean percentage (average of both eyes) of central and peripheral field efficiency compared with number of driving years for 42 retinitis pigmentosa patients

\begin{tabular}{llll}
\hline \multirow{2}{*}{ Sex } & \multicolumn{3}{l}{ Years driving, \% } \\
\cline { 2 - 4 } & $5-10$ & $10-20$ & 21 or more \\
\hline Central efficiency (males) & 82 & 87 & 82 \\
Central efficiency (females) & 86 & 90 & 97 \\
Peripheral efficiency (males) & 14 & 28 & 18 \\
Peripheral efficiency (females) & 21 & 26 & 55 \\
\hline
\end{tabular}


a driving hazard. ${ }^{9-11}$ Feinbloom ${ }^{9}$ noted that the use of the side-view mirror is almost totally dependent on the utilisation of the peripheral retina, and that a driver who is handicapped in that area would be expected to have difficulty perceiving the road both ahead and behind. Keeney ${ }^{12}$ concluded that from a practical point of view a horizontal field of vision of $140^{\circ}$ is needed to operate a motor vehicle safely. Nevertheless, the study of Danielson ${ }^{13}$ would suggest that defects in a driver's peripheral field of vision are not a significant factor in the cause of accidents. He states that the quality of the central field is apparently a more important factor in driving safety than the quantity of the peripheral field.

The variables responsible for causing an automobile accident are numerous. In addition to visual function they include, among others, driving speed, alertness and cautiousness, experience, use of alcohol or other drugs, age, sex, environment and familiarity with surroundings, condition of the automobile vehicle, and so on. This report attempts to assess the role of visual factors, particularly peripheral visual field, by comparing 2 populations: those with normal visual function and those with variable degrees of visual field and central visual loss. The findings suggest that, although RP patients appear to have a greater number of accidents than a control population of similar age, the higher rate was largely attributable to a subgroup of female RP patients who had a disproportionately high number of accidents in comparison with those of female control subjects, when those driving for 5 to 10 years were compared. Moreover, we were unable to find positive correlations between central acuity and peripheral field efficiency and driving performance.

The explanation for the better-than-expected results in RP patients reported in this preliminary study is uncertain. It seems relevant that $74 \%$ of the RP patients reported that they voluntarily restricted their driving to daylight hours, when fewer accidents tend to occur. ${ }^{14}$ Further studies are required to determine the importance of other variables (race, type and location of most frequent driving, self-imposed restrictions on driving) as they relate to safe driving by RP patients. Why the female subgroup of RP patients who drove 5 to 10 years were at greater risk of involvement in a driving accident is uncertain. Nevertheless, their disproportionately high number of accidents appears to account largely for the greater percentage of accidents experienced by the RP patients as a group.

This investigation was supported in part by core grant 1 P30EY01792 and by grant EY-703802 from the National Eye Institute, National Institutes of Health, Bethesda, Maryland; by an unrestricted grant and manpower award from Research to Prevent Blindness, Inc, NYC; and by a research grant from the National Retinitis Pigmentosa Foundation, Baltimore, Md.

\section{References}

1 Pearson RM. The objective determination of vision and vision acuity. Br J Physiol Opt 1966; 23: 107-28.

2 Bier N. Correction of Subnormal Vision. New York: Appleton-Century-Crofts, $1970 ; 151-2$.

3 National Safety Council. Accident Facts. Chicago: National Safety Council, 1978; 54.

4 Mantel N, Haenszel W. Statistical aspects of the analysis of data from retrospective studies of disease. J Natl Cancer Inst 1959; 22: 719-48.

5 Keeney AH. Field loss vs central magnification-telescopes and driving risk. Arch Ophthalmol 1974; 92: 273.

6 Newman JD. The rationale for licensure: Criteria and training. In: Faye EE, Hood CM, eds. Low Vision. Springfield: Thomas, $1975 ; 179-82$.

7 Keller JT, Eskridge JB. Telescopic lenses and driving. Am J Optom Physiol Opt 1976; 53: 746-9.

8 Newman JD. A rational approach to license drivers using bioptic telescopes. J Am Optom Assoc 1976; 47: 510-13.

9 Feinbloom W. Head and eye movements while driving. Read before the American Academy of Optometry, New York City, December 1972.

10 Committee on Medical Aspects of Automobile Safety. Visual factors in automobile driving, and provisional standards. Arch Ophthalmol 1969; 81 : 865-71.

11 Byrnes VA. Visual factors in automobile driving. Trans Am Ophthalmol Soc 1962; 60: 60-83.

12 Keeney AH. Ophthalmology in driving. Trans Pac Coast Otoophthalmol Soc 1967; 48: 167-78.

13 Danielson $\mathrm{RW}$. The relationship of fields of vision to safety in driving. Am J Ophtha!mol 1957; 44: 657-80.

14 Lebensohn JE. The seeing factors in traffic safety. Sight Sav Rev 1949; 19: 191-200. 